(2) Open Access Full Text Article

\title{
Analysis of Complications for Epiretinal Membrane and Macular Hole Surgery Performed by Vitreoretinal Fellows and Consultants
}

\author{
Saud Aljohani ${ }^{1,2}$ \\ Abdulaziz Alshehri ${ }^{1,3}$ \\ Abdulaziz Al Taisan (D) 1,4 \\ Rakan Algorinees ${ }^{1,5}$ \\ Valmore A Semidey (D) $^{\prime}$ \\ 'King Khaled Eye Specialist Hospital, \\ Riyadh, Kingdom of Saudi Arabia; ${ }^{2}$ King \\ Fahad University Hospital, Alkhobar, \\ Kingdom of Saudi Arabia; ${ }^{3}$ Altaif \\ University, Al-Taif, Saudi Arabia; ${ }^{4}$ King \\ Faisal University, Al-Ahsa, Saudi Arabia; \\ ${ }^{5}$ University of Ha'il, Ha'il, Saudi Arabia
}

Correspondence: Valmore A Semidey Vitreoretinal Division, King Khaled Eye Specialist Hospital, Al- Oruba Street, PO Box 7191, Riyadh, II462, Kingdom of Saudi Arabia

Tel +966 II 482 I 234 Ext. 2655

Fax +966 II $482 / 234$ Ext. 3773

Email asemidey@hotmail.com
Purpose: The aim of this study was to report the intraoperative and postoperative complications of vitrectomy for epiretinal membrane (ERM) and macular hole (MH) performed by retinal fellows under direct faculty supervision compared with experienced faculty members. Patients and Methods: A total of 271 eyes that underwent pars plana vitrectomy (PPV) for MH and ERM from January 2014 to December 2019 at King Khaled Eye Specialist Hospital were analyzed. PPV for ERM and MH was performed by vitreoretinal fellows and consultants.

Results: The outcome measures assessed were the intraoperative complications rates, such as posterior lens touch, retinal breaks (RBs), retinal detachments, and vitreous hemorrhage. Moreover, the postoperative complications and optical coherence tomography (OCT) changes were assessed upon a minimum follow-up of 6 months. The rate of iatrogenic RB was more common in the ERM than in the MH surgery (15.5\% vs $11.2 \%)$. Fellows and consultants had a rate of $20.5 \%$ of RB during the ERM surgery and $14.6 \%$ during $\mathrm{MH}$ surgery, respectively. However, these differences were not statistically significant $(p=0.12$ for $\mathrm{MH}$ and $p=0.236$ for ERM). Postoperative OCT analysis revealed an MH closure rate of $72.2 \%$, and complete removal of the ERM was achieved in $88.6 \%$ in cases performed by fellows, while consultants achieved $61.8 \%$ closure rate of $\mathrm{MH}$, and $83.3 \%$ of the patients achieved complete removal of ERM.

Conclusion: Macular surgery is overall a safe procedure and the complication rates between fellows and consultants are comparable. With proper supervision, vitreoretinal fellows can achieve equally high anatomical outcomes with few complications.

Keywords: epiretinal membrane, fellows, macular hole, pars plana vitrectomy, retinal break

\section{Introduction}

With the advent of small gauge vitrectomy systems, pars plana vitrectomy (PPV) has become the most common procedure performed by vitreoretinal in-training fellows. ${ }^{1}$ Similar to any other procedure, complications have been described, and they include cataract, high intraocular pressure (IOP), retinal vascular occlusion, intraocular inflammation, endophthalmitis, cystoid macular edema, phototoxicity, macular holes, retinal breaks (RBs), retinal detachment (RD), and surgery failures. The incidence of iatrogenic RBs is reported to be significantly higher in patients with idiopathic macular hole $(\mathrm{MH})$ than in those with an epiretinal membrane (ERM). ${ }^{2}$

The safety and efficacy of PPV performed by fellows are valuable points of interest and concern in educational institutions. The success rates and the rates of 
perioperative complications of in-training fellows and graduates play a crucial role in the quality and appeal of the training center.

The aim of this study was to report our institution's experience in intraoperative and postoperative complication rates for PPV in $\mathrm{MH}$ and ERM operations performed by the same group of vitreoretinal surgeons (consultants and fellows).

\section{Patients and Methods}

\section{Participants}

This study was carried out in adherence to the Declaration of Helsinki and was approved by the Institutional Review Board at King Khaled Eye Specialist Hospital in which the requirement for informed consent was waived due to the retrospective nature of the study. A total of 271 eyes that underwent PPV for MH and ERM from January 2014 to December 2019 at King Khaled Eye Specialist Hospital were analyzed. All eyes that underwent PPV for idiopathic full-thickness $\mathrm{MH}$ and ERM were included in the study; any patient with combined phacoemulsification and PPV, traumatic MH, previous PPV, proliferative diabetic retinopathy (PDR), age-related macular degeneration and previous macular laser therapy was excluded from this study.

\section{Testing}

A vitreoretinal fellow or consultant performed vitrectomy using the Constellation Vitrectomy System (ALCON, Ft. Worth, TX, USA) with standard 23- or 25-gauge PPV. Initially, a core vitrectomy was carried out followed by induction of posterior vitreous detachment, if not already present, with the assistance of intravitreal triamcinolone. The internal limiting membrane was removed using Eckardt or ILM forceps after staining with MembraneBlue-Dual (DORC International, The Netherlands) until reaching the vascular arcades, the infusion was turned off and the dye was left for 2 min and re-stained in case of any uncertainty upon proper removal. Technique for macular hole closure was left at surgeon's discretion, revising of the periphery and performing laser treatment of any RB. Air, sulphur hexafluoride (SF6), perfluoropropane (C3F8) or silicone oil were used as an intravitreal tamponade.

Data were collected from an electronic patient record system. It included laterality of the affected eye, indication of surgery (ERM vs $\mathrm{MH}$ ), patient age, diabetic status, and grade of the surgeon (fellow vs consultant). Consultants were considered to have a minimum of five years of surgical experience after completion of fellowship training. The Intraoperative complications were recorded; they included posterior lens touch, RB, RD, and vitreous hemorrhage (VH). Subsequently, postoperative clinical (VH, endophthalmitis, RD, persistent $\mathrm{MH}$ and the need for phacoemulsification surgery within 1 year) and optical coherence tomography (OCT) (persistent MH, residual ERM, localized retinal thinning, ellipsoid zone disruption, central macular thickness) complications were recorded with a minimum follow-up of 6 months. A highly skilled ocular photographer carried out the spectral domain (SD)-OCT (SPECTRALIS SD-OCT; Heidelberg Engineering, Heidelberg, Germany). To assess the macular area, the examiner obtained retinal images by performing $512 \times 128$ scan pattern where a $6 \times$ 6-mm-area on the retina was scanned using 128 horizontal and vertical lines, each consisting of 512 A-scans per line (total of 65,536 sampled points) within a scan time of $2.4 \mathrm{~s}$. Internal fixation was applied to ensure proper alignment of the eye. If the patient had poor fixation, based on the fundus image generated by the machine, the image on the macula could be centered by the photographer.

\section{Outcome Measures}

The primary outcome was the incidence of intraoperative and post-operative complications among consultants and fellows.

\section{Data Analysis}

Data were analyzed using the IBM SPSS software (IBM, Armonk, NY, USA). The continuous variables were presented as mean with standard deviations (SD) and the categorical variables as frequencies and percentages. The rates of the posterior segment complications were compared by the chi-square analysis between the ERMs and MHs, and the consultants and fellows. The multivariate logistic regression was performed to determine the risk factors associated with having one or more posterior segment intraoperative and postoperative complications, among the indications for surgery (ERM vs MH) and the grade of the surgeon (consultants vs fellows). The Chisquare test or the Fisher's exact test was used for the comparison of proportions such as RBs.

\section{Results}

The study included 271 operated eyes of 271 patients (141 [52\%] male and 130 [48\%] female) with an age of $59.15 \pm$ 18.11 years. Of these, 161 eyes $(59.4 \%)$ were operated for idiopathic MH and 110 eyes (40.5\%) for ERM. Patient demographics and preoperative ocular data can be seen in Table 1. 
Table I Patient Demographics, Preoperative Ocular Data and Tamponade Agents

\begin{tabular}{|c|c|c|c|c|c|c|c|c|}
\hline & & \multicolumn{6}{|c|}{ Pre-Operative Diagnosis } & \multirow[t]{3}{*}{$p$ value } \\
\hline & & \multicolumn{2}{|c|}{$M H(n=161)$} & \multicolumn{2}{|c|}{$\operatorname{ERM}(n=1 \mid 0)$} & \multicolumn{2}{|c|}{ Total $(n=27 I)$} & \\
\hline & & Freq & $\%$ & Freq & $\%$ & Freq & $\%$ & \\
\hline \multicolumn{2}{|l|}{ Age, years } & \multicolumn{2}{|c|}{$57.35 \pm 19.46$} & \multicolumn{2}{|c|}{$61.77 \pm 15.66$} & \multicolumn{2}{|c|}{$59.15 \pm 18.11$} & 0.470 \\
\hline \multirow[t]{2}{*}{ Gender } & Male & 69 & 42.9 & 72 & 65.5 & $|4|$ & 52.0 & $<0.001$ \\
\hline & Female & 92 & 57.1 & 38 & 34.5 & 130 & 48.0 & \\
\hline \multirow[t]{2}{*}{ Eye } & OD & 81 & 50.3 & 61 & 55.5 & 142 & 52.4 & 0.405 \\
\hline & OS & 80 & 49.7 & 49 & 44.5 & 129 & 47.6 & \\
\hline \multirow[t]{2}{*}{ Diabetes } & Yes & 58 & 36.0 & 77 & 70.0 & 135 & 49.8 & $<0.001$ \\
\hline & No & 103 & 64.0 & 33 & 30.0 & 136 & 50.2 & \\
\hline \multirow[t]{2}{*}{ Grade of Surgeon } & Consultant & 89 & 55.3 & 66 & 60.0 & 155 & 57.2 & $0.44 I$ \\
\hline & Fellow & 72 & 44.7 & 44 & 40.0 & 116 & 42.8 & \\
\hline \multirow[t]{3}{*}{ Lens status } & Phakic & 96 & 59.6 & 46 & 41.8 & 142 & 52.4 & 0.003 \\
\hline & Pseudophakic & 65 & 40.4 & 62 & 56.4 & 127 & 46.9 & \\
\hline & Aphakic & 0 & 0.0 & 2 & 1.8 & 2 & 0.7 & \\
\hline \multirow[t]{5}{*}{ Tamponade } & None & I & 0.6 & 48 & 43.6 & 49 & 18.1 & $<0.001$ \\
\hline & SF6 & 93 & 57.8 & 19 & 17.3 & 112 & 41.3 & \\
\hline & $\mathrm{C} 3 \mathrm{~F} 8$ & 46 & 28.6 & 9 & 8.2 & 55 & 20.3 & \\
\hline & Air & 3 & 1.9 & 28 & 25.5 & 31 & 11.4 & \\
\hline & Silicone Oil & 18 & 11.2 & 6 & 5.5 & 24 & 8.9 & \\
\hline
\end{tabular}

Abbreviations: MH, macular hole; ERM, epiretinal membrane; Freq, frequency; C3F8, perfluoropropane; SF6, sulphur hexafluoride.

Table 2 Intraoperative Complication Rates According to Surgical Indication

\begin{tabular}{|c|c|c|c|c|c|c|c|c|}
\hline & & \multicolumn{6}{|c|}{ Pre-Operative Diagnosis } & \multirow[t]{3}{*}{$p$ value } \\
\hline & & \multicolumn{2}{|c|}{$M H(n=161)$} & \multicolumn{2}{|c|}{$\operatorname{ERM}(n=|| 0)$} & \multicolumn{2}{|c|}{ Total $(n=27 I)$} & \\
\hline & & Freq & $\%$ & Freq & $\%$ & Freq & $\%$ & \\
\hline \multirow[t]{2}{*}{ Lens touch } & No & 159 & 98.8 & 110 & 100.0 & 269 & 99.3 & 0.516 \\
\hline & Yes & 2 & 1.2 & 0 & 0.0 & 2 & 0.7 & \\
\hline \multirow[t]{2}{*}{ Retinal breaks } & No & 143 & 88.8 & 93 & 84.5 & 236 & 87.1 & 0.303 \\
\hline & Yes & 18 & 11.2 & 17 & 15.5 & 35 & 12.9 & \\
\hline \multirow[t]{2}{*}{ Retinal Detachment } & No & 161 & 100.0 & 107 & 97.3 & 268 & 98.9 & 0.066 \\
\hline & Yes & 0 & 0.0 & 3 & 2.7 & 3 & 1.1 & \\
\hline
\end{tabular}

Abbreviations: $\mathrm{MH}$, macular hole; ERM, epiretinal membrane; Freq, frequency.

Lens touch rate was $1.2 \%$ overall in the series, which was not found to be statistically different across any of the groups compared. Regarding posterior segment complications, the only statistically significant difference across the groups occurred in the rates of RBs, which were more common in ERM (15.5\%) than in MH (11.2\%) (Table 2). Consultants had a higher number of RBs during $\mathrm{MH}$ surgery $(14.6 \%)$ in comparison to $(6.9 \%)$ for the cases done by the fellows, whereas fellows had more RBs during
ERM surgery (20.5\%) and consultants (12.1\%). However, these differences were not statistically significant $(p=$ 0.125 for $\mathrm{MH}$ and 0.236 for ERM) (Table 3). Consultants performed more surgeries than fellows did, but the proportion of overall RBs was similar for both $\mathrm{MH}$ and ERM across both groups (13.5\% and $12.1 \%$, respectively). Other factors, including the patient's age, gender, and diabetes, were not significant. The rate of intraoperative RD was observed in only three cases of 
Table 3 Intraoperative Breakdown of Retinal Breaks by Surgeon Grade

\begin{tabular}{|c|c|c|c|c|c|c|c|c|c|c|c|c|c|}
\hline & & \multicolumn{12}{|c|}{ Pre-Operative Diagnosis } \\
\hline & & \multicolumn{4}{|c|}{$M H(n=161)$} & \multicolumn{4}{|c|}{$\operatorname{ERM}(n=|| 0)$} & \multicolumn{4}{|c|}{ Total $(n=27 I)$} \\
\hline & & \multicolumn{2}{|c|}{ Consultant } & \multicolumn{2}{|c|}{ Fellow } & \multicolumn{2}{|c|}{ Consultant } & \multicolumn{2}{|c|}{ Fellow } & \multicolumn{2}{|c|}{ Consultant } & \multicolumn{2}{|c|}{ Fellow } \\
\hline & & Count & $\%$ & Count & $\%$ & Count & $\%$ & Count & $\%$ & Count & $\%$ & Count & $\%$ \\
\hline \multirow[t]{2}{*}{ Retinal breaks } & No & 76 & 85.4 & 67 & 93.1 & 58 & 87.9 & 35 & 79.5 & 134 & 86.5 & 102 & 87.9 \\
\hline & Yes & 13 & 14.6 & 5 & 6.9 & 8 & 12.1 & 9 & 20.5 & 21 & 13.5 & 14 & 12.1 \\
\hline
\end{tabular}

Abbreviations: $\mathrm{MH}$, macular hole; ERM, epiretinal membrane.

ERM, and this result was only marginally significant $(p=0.066)$

Patients had a minimum follow-up of 6 months (range 6-48 months). The total postoperative closure rate for $\mathrm{MH}$ was $66.4 \%$, which was higher in the cases performed by fellows than in those performed by consultants $(72.2 \%$ and $61.8 \%$, respectively). While persistent $\mathrm{MH}$ was observed in $33.6 \%$ of all cases, the persistent $\mathrm{MH}$ rate was higher in the cases performed by consultants than in those performed by fellows (38.2\% and $27.8 \%$, respectively). Compared to $\mathrm{MH}$, ERM had a higher success rate of $85.4 \%$ in terms of total removal of the ERM. Cases performed by fellows had a higher success rate of $88.6 \%$ than those performed by consultants, which amounted to $83.3 \%$. Other complications assessed were localized retinal thinning, which occurred in $25.8 \%$ of all cases, and was observed more in the cases with
ERM. Ellipsoid zone disruption was similar between the groups (20.4\% and 17.2\% for MH and ERM, respectively). Moreover, the rate of thickened macular area (persistent macular edema) with loss of foveal depression was observed in $20.9 \%$ (23/110 eyes) of the patients with ERM (Table 4). Based on the need for phacoemulsification surgery within 12 months, the cataract progression rate assessed amounted to be $22.5 \%$ across all cases. Cases with ERM were more likely to require cataract surgery compared to $\mathrm{MH}(26.0 \%$ vs $20.8 \%$ ). At the last follow-up, no cases of endophthalmitis and two cases of $\mathrm{VH}$ were documented.

\section{Discussion}

Several previous reports have compared the rate of complications in vitreoretinal surgery between in-training staff and established faculty. ${ }^{1,3}$ However, we focused mainly on

Table 4 Post-Operative OCT Changes

\begin{tabular}{|c|c|c|c|c|c|c|c|c|c|c|c|c|c|c|c|}
\hline \multirow[t]{4}{*}{ OCT Changes } & \multicolumn{12}{|c|}{ Pre-Operative Diagnosis } & & & \\
\hline & \multicolumn{4}{|c|}{$M H(n=161)$} & \multicolumn{4}{|c|}{$\operatorname{ERM}(n=|| 0)$} & \multicolumn{4}{|c|}{ Total $(n=27 I)$} & \multicolumn{3}{|c|}{ Total } \\
\hline & \multicolumn{2}{|c|}{$\begin{array}{c}\text { Consultant } \\
(n=89)\end{array}$} & \multicolumn{2}{|c|}{$\begin{array}{l}\text { Fellow } \\
(n=72)\end{array}$} & \multicolumn{2}{|c|}{$\begin{array}{c}\text { Consultant } \\
(n=66)\end{array}$} & \multicolumn{2}{|c|}{$\begin{array}{l}\text { Fellow } \\
(n=44)\end{array}$} & \multicolumn{2}{|c|}{$\begin{array}{c}\text { Consultant } \\
(n=\mid 55)\end{array}$} & \multicolumn{2}{|c|}{$\begin{array}{c}\text { Fellow } \\
(n=1 \mid 6)\end{array}$} & & & \\
\hline & Freq & $\%$ & Freq & $\%$ & Freq & $\%$ & Freq & $\%$ & Freq & $\%$ & Freq & $\%$ & & & \\
\hline Closed MH & 55 & 61.8 & 52 & 72.2 & 0 & 0.0 & 0 & 0.0 & 55 & 35.5 & 52 & 44.8 & & $66.4 \%$ & \\
\hline Persistent $\mathrm{MH}$ & 34 & 38.2 & 20 & 27.8 & 0 & 0.0 & 0 & 0.0 & 34 & 21.9 & 20 & 17.2 & & $33.6 \%$ & \\
\hline No ERM & 0 & 0.0 & 0 & 0.0 & 55 & 83.3 & 39 & 88.6 & 55 & 35.5 & 39 & 33.6 & & $85.4 \%$ & \\
\hline Persistent ERM & 0 & 0.0 & 0 & 0.0 & 11 & 16.7 & 5 & 11.4 & II & 7.1 & 5 & 4.3 & & $14.6 \%$ & \\
\hline $\begin{array}{l}\text { Localized retinal } \\
\text { thinning }\end{array}$ & 18 & 20.2 & 16 & 22.2 & 21 & 31.8 & 15 & 34.1 & 39 & 25.2 & 31 & 26.7 & $\begin{array}{c}\mathrm{MH} \\
21.1 \%\end{array}$ & $\begin{array}{l}\text { ERM } \\
32.6 \%\end{array}$ & $\begin{array}{c}\text { ALL } \\
25.8 \%\end{array}$ \\
\hline EZ disruption & 16 & 18.0 & 17 & 23.6 & 10 & 15.2 & 9 & 20.5 & 26 & 16.8 & 26 & 22.4 & $\begin{array}{c}\mathrm{MH} \\
20.4 \%\end{array}$ & $\begin{array}{c}\text { ERM } \\
17.2 \%\end{array}$ & $\begin{array}{c}\text { ALL } \\
19.1 \%\end{array}$ \\
\hline Persistent CME & I & 1.1 & 1 & 1.4 & 15 & 22.7 & 8 & 18.2 & 16 & 10.3 & 9 & 7.8 & $\begin{array}{l}\mathrm{MH} \\
1.2 \%\end{array}$ & $\begin{array}{l}\text { ERM } \\
20.9 \%\end{array}$ & $\begin{array}{l}\text { ALL } \\
9.2 \%\end{array}$ \\
\hline
\end{tabular}

Abbreviations: $\mathrm{MH}$, macular hole; ERM, epiretinal membrane; OCT, optical coherence tomography; EZ, ellipsoid zone; CME, cystoid macular edema. 
macular surgery, which is assumed to have a steeper learning curve. Our study simulates similar complication rates between these groups, with the most common intraoperative complication being the development of RBs (12.9\% overall).

Despite advances in vitreoretinal surgery in recent years, including the advent of small gauge cannulated systems, complications can occur even at the hands of experienced vitreoretinal surgeons. ${ }^{3}$

The anterior segment intraoperative complications were assessed in our series in phakic patients (142 eyes). Lens touch is one of the causes for cataract formation, which appears as a line on the posterior surface of the lens capsule perioperatively. This complication has the potential for cataract formation in the postoperative period, thereby requiring further surgery for correction. Our results are consistent with those of Jackson et al, ${ }^{4}$ who found the rate of lens touch to be $1.2-1.6 \%$ in PPVs. Cataract progression in patients with phakic lenses requiring surgery over a 12 months' period was $22.5 \%$, consistent with Kataria et $\mathrm{al}^{5}{ }^{5}$ who also found the cataract progression rate to be $34.6 \%$ following PPV, highlighting the long-term effect of tamponade agents used during these procedures.

Analysis of the intraoperative posterior segment complications (Table 2) revealed that RBs were more common in ERM than in $\mathrm{MH}$. Chung et $\mathrm{al}^{6}$ reported an incidence of $14.6 \%$ iatrogenic $\mathrm{RBs}$ in $\mathrm{MH}$ surgeries, which was higher than our results $(11.2 \%)$. In contrast, rates of RBs in ERM surgeries were significantly lower in their study than in ours $(6.9 \%$ versus $15.5 \%)$. It could be postulated that in our series $70 \%$ (77/110 eyes) of patients with ERM had diabetes, anddespite proliferative diabetic retinopathy being excluded from our series, they could present with a more adherent posterior vitreous than the patients without diabetes, which plays an important role in posterior vitreous detachment (PVD) induction. ${ }^{7}$ Moreover, patients with diabetes may have small inadvertent peripheral neovascularization with an adherent peripheral hyaloid that may lead to a break during a PVD induction. Additionally, Yagi et $\mathrm{al}^{8}$ reported a higher rate of iatrogenic RBs of 35.4\% (28/79 eyes) associated with $\mathrm{MH}$ surgery when compared to ERM.

Our study also revealed that the rate of RBs during $\mathrm{MH}$ surgery for consultants was $14.6 \%$ (13/89 eyes), which is lower than that reported in the literature. ${ }^{8}$ On the other hand, fellows had fewer MH related RBs (6.9\%; 5/72 eyes), which may be due to multiple factors. One possible explanation is that the less experienced trainee worked on more straightforward or less complicated PPV cases. Our training center is also equipped with a virtual reality simulator in which fellows can undergo basic training during their initial months, which allows them to be accustomed to microforceps handling and tremor control. In addition, it is likely that heavy supervision by the attending allowed for fewer complications. On the other hand, more experienced staff will operate on advanced $\mathrm{MH}$ cases (myopic $\mathrm{MH}$ ), where strongly adherent hyaloid in midperiphery could lead to RB formation. ERM fellows' surgeries were associated with a higher rate of $\mathrm{RBs}(20.5 \%)$ compared to that published in the literature. ${ }^{6,8}$ This could be explained by the lack of experience and attention being paid to the periphery during PVD induction. This highlights the fact that ERM development is an alteration of the vitreoretinal interphase, and its pathology and strong adhesion are not limited to the macular area but could involve the mid and far periphery of the retina as well.

In recent years, refinements in surgical techniques, visualization, and staining agents have been associated with excellent results in terms of $\mathrm{MH}$ closure. Across many studies, surgical success assessed postoperatively with OCT revealed consistently high primary closure rates $(>85 \%)$; factors associated with a higher success rate were smaller $\mathrm{MH}$ and shorter duration. ${ }^{9-11}$ However, since our institution is a tertiary referral center and patients usually presented with chronic or large $\mathrm{MH}$, the total rate of $\mathrm{MH}$ closure was $66.4 \%$. Our study also assessed the ERM persistence rate, and it was $14.6 \%$ (15/110 eyes) in comparison to Schechet et $\mathrm{al}^{12}$ who found the ERM persistent rate in eyes that did not undergo ILM peeling to be $22.9 \%$. This finding could be explained by our routine use of Dual Blue and double staining, which allows for proper primary removal of the ERM and simultaneous ERM/ILM peeling during the same procedure.

Surgically included trauma (localized retinal thinning $25.8 \%$ ) or chronicity and natural course of the macular pathology (ellipsoid zone disruption 19.1\% and persistent CME 9.2\%) could be responsible for the ultrastructural OCT changes in the postoperative period evaluated in our study. Cystoid macular edema resolution with normalization of the foveal depression in the postoperative period was higher in the $\mathrm{MH}$ group (98.2\%) than in the ERM group (79.1\%). This result was lower than that reported by Lee et al, ${ }^{13}$ who showed an $84.2 \%$ rate of normal foveal contour with a foveal depression in cases of ERM peeling.

Our study had certain limitations. First, it was a retrospective study and showed a lack of correlation between documented complications and visual outcomes in the pre- and postoperative periods. However, our 
sample size was larger than that of previous studies comparing fellows and attending complications in macular surgery (271 patients vs 232 in Mason et al). ${ }^{1}$ Further prospective studies, especially with the availability of better viewing systems and instrumentation, correlating the visual outcomes of intra and postoperative complications between fellows and consultants are warranted.

\section{Conclusion}

Macular surgery is overall a safe procedure, and complication rates between fellows and consultants are comparable. Considering that the most common complications (RBs) arise from the nature of the pathology at the vitreoretinal interphase itself, careful supervision, better visualization systems, and use of vital dyes can further reduce the risk of complications to achieve anatomical success.

\section{Abbreviations}

ERM, epiretinal membrane; FTMH, full-thickness macular hole; ILM; internal limiting membrane; IOP, intraocular pressure; $\mathrm{MH}$, macular hole; OCT, optical coherence tomography; PPV, pars plana vitrectomy; PVD, posterior vitreous detachment; RB, retinal breaks; RD, retinal detachments; $\mathrm{SD}$, standard deviation; $\mathrm{VH}$, vitreous hemorrhage.

\section{Data Sharing Statement}

The datasets used and/or analysed during the current study are available from the corresponding author on reasonable request.

\section{Ethics Approval and Informed Consent}

This study was carried out in adherence to the Declaration of Helsinki and was approved by the Institutional Review Board at King Khaled Eye Specialist Hospital in which the requirement for informed consent was waived due to the retrospective nature of the study.

\section{Consent for Publication}

Written informed consent for publication was not required due to the retrospective nature of the study and the lack of identifiable patient data.

\section{Acknowledgments}

We would like to acknowledge our biostatistician, Dr. Syed Khabir Ahmad, for his contribution to this project.

\section{Author Contributions}

All authors made a significant contribution to the work reported, whether that is in the conception, study design, execution, acquisition of data, analysis and interpretation, or in all these areas; took part in drafting, revising or critically reviewing the article; gave final approval of the version to be published; have agreed on the journal to which the article has been submitted; and agree to be accountable for all aspects of the work.

\section{Funding}

No funding was used for the design and conduction of this study.

\section{Disclosure}

The authors have no proprietary, commercial, or noncommercial interest in any materials mentioned in this article. The authors report no conflicts of interest in this work.

\section{References}

1. Mason JO, Mason LB, Patel SA, et al. Vitreoretinal surgical outcomes performed by supervised retinal fellows versus attending faculty surgeons. Retina. 2016;36:981-985. doi:10.1097/IAE.000 0000000000828

2. Guillaubey A, Malvitte L, Lafontaine PO, et al. Incidence of retinal detachment after macular surgery: a retrospective study of 634 cases. Br J Ophthalmol. 2007;91:1327-1330. doi:10.1136/bjo.2007.115162

3. Wilkinson JT, Richards AB, Choi D, Robertson JE, Flaxel CJ. Incidence of retinal detachment after fellow-performed primary pars plana vitrectomy. ISRN Ophthalmol. 2013;19:353209.

4. Jackson TL, Donachie PH, Sparrow JM, Johnston RL. United Kingdom national ophthalmology database study of vitreoretinal surgery: report 1; case mix, complications, and cataract. Eye (Lond). 2013;27:644-651. doi:10.1038/eye.2013.12

5. Kataria AS, Thompson JT. Cataract formation and progression in patients less than 50 years of age after vitrectomy. Ophthalmol Retina. 2007;1:149-153. doi:10.1016/j.oret.2016.09.007

6. Chung SE, Kim KH, Kang SW. Retinal breaks associated with the induction of posterior vitreous detachment. Am J Ophthalmol. 2009;147:1012-1016. doi:10.1016/j.ajo.2009.01.013

7. Nesmith BL, Palacio AC, Schaal Y, Gupta A, Schaal S. Diabetes alters the magnitude of vitreomacular adhesion. Retina. 2017;37:749-752. doi:10.1097/IAE.0000000000001228

8. Yagi F, Takagi S, Tomita G. Incidence and causes of iatrogenic retinal breaks in idiopathic macular hole and epiretinal membrane. Semin Ophthalmol. 2014;29:66-69. doi:10.3109/08820538.2012.760627

9. Essex RW, Kingston ZS, Moreno-Betancur M, et al. The effect of postoperative face-down positioning and of long- versus short-acting gas in macular hole surgery: results of a registry-based study. Ophthalmology. 2016;123:1129-1136. doi:10.1016/j.ophtha.2015. 12.039

10. Muselier A, Dugas B, Burelle X, et al. Macular hole surgery and cataract extraction: combined vs consecutive surgery. $\mathrm{Am}$ J Ophthalmol. 2010;150:387-391. doi:10.1016/j.ajo.2010.04.008

11. Lois N, Burr J, Norrie J, et al. Internal limiting membrane peeling versus no peeling for idiopathic full-thickness macular hole: a pragmatic randomized controlled trial. Invest Ophthalmol Vis Sci. 2011;52:1586-1592. doi:10.1167/iovs.10-6287 
12. Schechet SA, DeVience E, Thompson JT. The effect of internal limiting membrane peeling on idiopathic epiretinal membrane surgery, with a review of the literature. Retina. 2017;37:873-880. doi:10.1097/IAE.0000000000001263
13. Lee JW, Kim IT. Outcomes of idiopathic macular epiretinal membrane removal with and without internal limiting membrane peeling: a comparative study. Jpn $J$ Ophthalmol. 2010;54:129-134. doi:10.1007/s10384-009-0778-0

\section{Publish your work in this journal}

Clinical Ophthalmology is an international, peer-reviewed journal covering all subspecialties within ophthalmology. Key topics include: Optometry; Visual science; Pharmacology and drug therapy in eye diseases; Basic Sciences; Primary and Secondary eye care; Patient Safety and Quality of Care Improvements. This journal is indexed on PubMed

Submit your manuscript here: https://www.dovepress.com/clinical-ophthalmology-journal
Central and CAS, and is the official journal of The Society of Clinical Ophthalmology (SCO). The manuscript management system is completely online and includes a very quick and fair peer-review system, which is all easy to use. Visit http://www.dovepress.com/ testimonials.php to read real quotes from published authors. 